\title{
Policy-Based Approach to Emergency Bio-Data Management for Mobile Healthcare
}

\author{
Seung-Man Chun and Jong-Tae Park* \\ School of Electronics Engineering, \\ Kyungpook National University, Daegu, Korea
}

Submitted July 2013. Accepted for publication February 2014.

\begin{abstract}
In m-healthcare service, accurate detection and notification of emergency situation are critical to chronic patients' life. Since they are usually performed by a limited number of medical staff, it is difficult to simultaneously support many patients in real-time. This article presents an architecture to support the emergency bio-data management for m-healthcare service using personalized emergency policy. The salient feature of the proposed architecture is that the decision on emergency is made using personalized emergency policy. Specifically, the structure of the detailed system components has also been designed. The emergency condition of the individual bio-data collected from wireless body area network is detected automatically using personalized emergency policy. The message flow diagram based on the personalized emergency policy is described. This enables quick emergency rescue service provided to the patient both accurately and immediately. The prototype of proposed system has been built to demonstrate the design concept.
\end{abstract}

Keywords: ISO/IEEE 11073 PHD, auto-detection management, personal health management, mobile healthcare

\section{INTRODUCTION}

Since mobile wireless pervasive computing and communication environments are changing, medical staff has new ways to interact with their patients and the elderly. By employing self-organized, wireless, physiology-monitoring hardware and software systems, continuous patient monitoring can assure timely intervention by a healthcare practitioner or physician. For example, cardiac patients wearing electrocardiogram (ECG) sensor systems can be monitored remotely without visiting the hospital. Healthcare monitoring systems are required to be connected to the Internet to transmit the bio-data in real time, at all times.

Usually, physiologic records are collected over a long period of time in order to allow accurate diagnoses and correct treatment by physicians. However, developing a pervasive sensor network for mobile healthcare (m-healthcare) service brings numerous challenges, including accurate collection techniques for bio-data

\footnotetext{
* Corresponding author: Jong-Tae Park, School of Electronics Engineering, Kyungpook National University,
} Daegu, Korea Phone: +82- 10-9805-5543. E-mail: jtpark@ee.knu.ac.kr. Other authors: smchun@ee.knu.ac.kr. 
information from the human body, seamless transmission techniques in various wireless networks, and development of healthcare applications. Patient-related biodata such as weight, ECG and electroencephalography (EEG) readouts, oxygen saturation $\left(\mathrm{SpO}_{2}\right)$, etc., measured by sensors in, over, on, and around the patient, are delivered to the medical staff or practitioner who checks the health status of the patient. In the delivery of patient-related data, international standards such as ISO/IEEE 11073 personalized health device (PHD) [1] and Health Level 7 clinical document architecture (HL7 CDA) [2] have been utilized to enable communication between medical, healthcare and wellness devices and external computer systems.

In a mobile healthcare monitoring service, accurate and prompt emergency detection and notification are very important [3]. However, in the current mobile healthcare service, it is difficult to provide in real time an accurate diagnosis of the patient due to a shortage in the number of medical practitioners [3]. As the bio-data measured from wireless body area network (WBAN) devices is usually transmitted to a remote physician through wireless communications technology, the bio-data may be lost or delayed due to unreliable wireless characteristics. In that case, the remote physician may not be able to detect or diagnose emergency status in the patient. Furthermore, because the critical level of certain bio-data pertaining to emergency might be different for each patient, an emergency decision based solely on the statistical level of the bio-data may be incorrect. For example, the emergency judgment from the bio-data might differ depending on the health characteristics of each person, such as age, history of disease, gender, etc. [4, 5, 6, 7]. Specifically, ECG is an important routine clinical practice for continuous monitoring of cardiac activities. However, ECG signals vary greatly for different individuals and within patient group [6]. Even for the same individual, heartbeat patterns significantly change with time and under different physical conditions. Since reliability of the health monitoring service will be degraded from an incorrect decision as to emergency, the decision about any emergency is very important.

In order to overcome the above-mentioned problems, we present an architecture of emergency bio-data management using a personalized emergency policy for mobile healthcare. More specifically, we have designed the management architecture using a personalized emergency policy for mobile healthcare. The structure of the detailed system components has also been designed, and the message flow diagram based on the personalized emergency policy is described. Finally, the implementation of the proposed system is presented.

Compared to other related works, the originality of our approach is described as follows:

- In order to judge an emergency decision from the bio-data measured by the body sensor, a personalized emergency policy is utilized. The personalized emergency policy is based on the critical-level information of the bio-data according to individual health conditions, such as age, sex, medical history, etc.

- In order to diagnose an emergency condition immediately, the health condition of the patient or person is tested and managed directly by a personalized smartphone, rather than a medical server system. 
- $\quad$ Architecture of message conversion between ISO/IEEE 11073 PHD and HL7 CDA is developed to efficiently transmit the bio-data from the personalized domain to the medical staff domain.

The proposed policy-based approach to emergency bio-data management consists of three steps. In step 1, the bio-data are collected by a wireless body area network. In step 2, the emergency decision is made using a personalized emergency policy. In step 3, the emergency message, including health information, is converted from an ISO/IEEE 11073 PHD message to HL7 CDA. By doing this, an emergency decision based on bio-data collected by the WBAN can be automatically made using a personalized emergency policy with smartphone. If emergency-level bio-data are detected, the ISO/IEEE 11073 agent in the smartphone transmits the emergency biodata to the ISO/IEEE 11073 PHD to HL7 CDA conversion server. Finally, the emergency bio-data are transmitted to the physician.

The rest of the article is structured as follows. In Section 2, we present the background on general mobile healthcare system. In Section 3, we describe our policy-based emergency bio-data management system for the mobile healthcare service. The detailed components of the proposed architecture using the personalized emergency policy are also described. In Section 4, the implementation details of the proposed system are described with service demonstration. Finally, the conclusion follows in Section 5.

\section{BACKGROUND ON MOBILE HEALTHCARE SYSTEM}

In this section, we address the generic architecture for a healthcare system. The general service architecture of the patient health monitoring system mainly consists of three domains: the body area, communications and networking, and health services. The body area domain is defined by wireless body area networks. A WBAN is composed of a number of sensors (e.g., sensor nodes such as an ECG monitor, pulse oximeter, blood pressure monitor) and a gateway (e.g., a broker gateway, smartphone or a notebook). The body sensors can be placed in, on, or around a patient's body to continuously monitor physiologic conditions such as heart rate and body temperature. They transmit bio-data to the gateway via wireless or wired network technologies such as IrDA cable, Bluetooth, ZigBee, and Wireless USB. The body sensor modes may be configured in a star topology or mesh. The major functionality of the communication and networking domain is to bridge the body area and service domains. Advanced wireless communications technologies such as cellular networks, $\mathrm{WiFi}$, and LTE may link WBAN gateways to the Internet and enable efficient mutual data communication between two WBANs. In the service domain, a trusted authority maintains an online server which is responsible for receiving and recording data in an electronic health record (EHR) or personalized health record (PHR), and analyzing the health-related information provided by medical staff or the health monitoring service provider. Finally, healthcare services such as health monitoring services, emergency rescue services, medical services, etc. can be offered. Most of all, the emergency rescue service is very important. Patient health information in a remote 
home can be monitored and managed by medical staff. However, in practice, it is difficult for the medical staff or emergency rescue provider to monitor or manage the patient health information. Specifically, as described in Section 1, since emergency judgment based on the bio-data might be different for each individual, it is difficult to provide real-time diagnosis for the patient due to a shortage of healthcare practitioners.

There are several research challenges associated with realizing effective mobile healthcare service. Some progresses have been made on international message standards such as ISO/IEEE 11073 PHD and HL7 CDA by the IEEE (Institute of Electrical and Electronics Engineers), ISO (International Standards Organization), HL7 (Health Level 7), etc. The ISO/IEEE 11073 standard is a family of standards to allow medical devices (i.e., the ISO/IEEE 11073 agent and manager) to interconnect and interoperate with each other. The framework of ISO/IEEE 11073 PHD consists of the domain information model (DIM), service model, and communication model. The ISO/IEEE 11073 agent may be implemented at the sensor node equipped with ISO/IEEE 11073 PHD agent protocol specifications, and the ISO/IEEE 11073 manager may be implemented at the smart mobile device equipped with the ISO/IEEE 11073 PHD agent protocol specifications. The ISO/IEEE 11073-20601 [1] optimized exchange protocol provides a framework of objectoriented information modeling, information access and measurement data transfer suitable for a wide variety of personalized health devices. The goal of IEEE 11072-20601 is to enable interoperability between sensors and data management devices to process, display or transfer the specific measurements.

The HL7 CDA is a document markup standard that specifies the structure and semantics of clinical documents for the purpose of exchange between healthcare providers and patients [2]. HL7 CDA conforms to the HL7 V3 Implementation Technology Specification (ITS), is based on the HL7 Reference Information Model (RIM), and uses HL7 V3 data types. It defines a clinical document having the characteristics such as persistence, stewardship, authentication, etc. HL7 CDA supports the exchange of clinical documents between those involved in the care of a patient, and the re-use of clinical data for public health reporting and quality monitoring. In order to enable the bio-data measured in the personalized home to be transmitted to clinical staff for diagnosing and monitoring the health condition or to make an emergency decision in real time, it is necessary to convert the message format of the bio-data based on ISO/IEEE 11073 PHD to the HL7 CDA message format.

There have been some research works on integrating ISO/IEEE 11073 and HL7 [8, $9,10,11,12,13]$. The relationships between the ISO/IEEE 11073 PHD model for ECG devices and the fields of the ISO 11073-91064 SCP-ECG standard were investigated [8]. Yao et al. [9] employed IEEE/ISO 11073 and Bluetooth standards to achieve device auto-association, interoperability, and simple reconfigurability. Mense et al. have presented a framework for transferring health data based on international standards [10]. The system is based on ISO/IEEE 11073 for device communication, CEN (European Committee for Standardization) 13606 for domain modeling, and HL7 
(Health Level 7) and IHE (Integrating the Healthcare Enterprise) profiles for communication with healthcare information systems and data storage. Yuksel et al. presented the HL7 RMIM of medical device domain from HL7 RIM based on IEEE 11073 DIM [11]. This makes it possible to trace the medical device data back to a standard common denominator. Yang et al. reported conversion from uCARDEA CIED to HL7 v2.x [12]. The approach presented by Yuksel et al. [11] is conceptually similar to the conversion from ISO/IEEE 11073 to HL7 message format, which is used in our approach. However, we have further developed the conversion from HL7 to ISO/IEEE 11073 message format.

ETRI [13] developed a home healthcare set-top box using the ISO/IEEE 11073 PHD standard. The home healthcare set-top box collects the bio-data according to the ISO/IEEE 11073 PHD standard, and provides a chronic-care service based on the collected bio-data. However, since the home healthcare set-top box is located in the home, the user or the patient can receive the health service only at home. In mobile healthcare, the bio-data of the patient should be monitored in real-time, anywhere. However, the home healthcare set-top box cannot support health service outside the home. In order to solve this limitations, the following additional research efforts are required for efficient mobile healthcare services: 1) the development of an automatic diagnosis mechanism based on personalized characteristics, 2) the efficient methods to convert the ISO/IEEE 11073 PHD standard and HL7 CDA, and vice versa, and 3) highly reliable emergency bio-data transmission over the wireless Internet.

\section{EMERGENCY BIO-DATA MANAGEMENT ARCHITECTURE USING PERSONALIZED EMERGENCY POLICY}

In this section, we describe the architecture for emergency bio-data management based on a personalized emergency policy. The detailed components of the proposed architecture using the personalized emergency policy are also described.

\subsection{Configuration of Emergency Management System using Personalized Emergency Policy}

Figure 1 depicts the configuration of the emergency management system using a personalized emergency policy for mobile healthcare service. The component in the configuration consists of the ISO/IEEE 11073 agent, ISO/IEEE 11073 manager, the personalized emergency policy database, the emergency management server (EMS), the hospital system, and the EHR/PHR database. The ISO/IEEE 11073 agent and manager are equipped with the ISO/IEEE 11073 PHD standard protocol stack. The ISO/IEEE 11073 agent collects bio-data such as a pulse oximeter, glucose meter, etc. The ISO/IEEE 11073 manager may be implemented on a smartphone, tablet PC, laptop PC, broker gateway, etc. The personalized emergency policy database is the database server that stores the critical values of the bio-data for a patient, as well as user authentication information. The critical values are used to judge whether the bio-data indicates an emergency or not, as the ISO/IEEE 11073 manager compares 


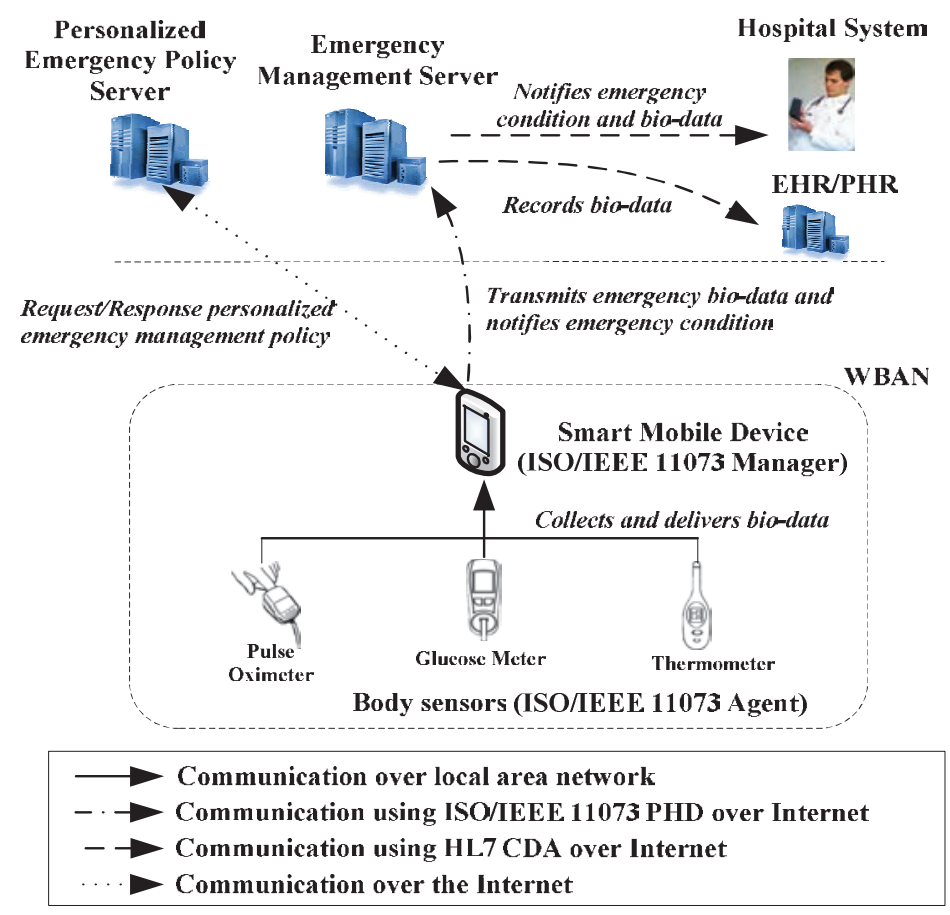

Figure 1. Configuration of emergency management using personalized emergency policy.

them with the critical values. The EHR/PHR is the database that stores the health record in digital format about individual patients or populations. The EHR may include a range of data, e.g., general demographics, medical history, medication and allergies, immunization status, laboratory test results, radiology images, vital signs, and personalized statistics such as age and weight. The EMS is a database server that performs message format conversion between the ISO/IEEE 11073 PHD and HL7 CDA standards to transmit in real time the bio-data from the remote user location to the medical staff.

The mobile healthcare service provided by the proposed configuration in Figure 1 consists of three steps: In step 1, the bio-data are collected by various body sensors, and are transmitted to the gateway through a WBAN. These body sensors may include the ISO/IEEE 11073 agents, and the gateway may include the ISO/IEEE 11073 manager. ISO/IEEE 11073-20601 standard can be employed to enable communication between the ISO/IEEE 11073 agent and ISO/IEEE 11073 manager. A wired/wireless network interface such as a wireless personal area network, local area network (LAN), or wireless LAN may be also used to exchange the bio-data between the ISO/IEEE 11073 agents and manager. 
In step 2, emergency decisions are made using a personalized emergency policy. When the ISO/IEEE 11073 manager at the gateway receives the bio-data through the ISO/IEEE 11073 agent at body sensors, it finds the emergency situation by utilizing the personalized policy information which is provided by the emergency policy database server. Usually, the ISO/IEEE 11073 manager stores and maintains the personalized emergency policy information in a local database and can judge immediately the emergency occurrences without the remote database access delay. In step 3, when the emergency situation is detected, the ISO/IEEE 11073 manager immediately delivers the level of emergency status with emergency bio-data to the emergency management server (EMS) in order to inform the medical staff of the emergency. This requires the message conversion between ISO/IEEE 11073 PHD and HL7 CDA. The EMS performs the conversion from the ISO/IEEE 11073 PHD message format to the HL7 CDA message format to enable the medical staff to accurately diagnose the individual bio-data.

With these three steps executed, the emergency situation can be detected both accurately and automatically, and relevant medical staff or physicians can be promptly notified, using a personalized emergency policy. Furthermore, in detecting and processing the emergency bio-data, the international standards such as ISO/IEEE 11073-20601 standard and the ISO/IEEE 11073 PHD/HL7 CDA conversion methods are employed, so that the emergency bio-data can be delivered to the medical staff through the existing medical information system in the hospital. This enhances both the applicability and efficiency of the proposed approach.

\subsection{Architecture of Smart Mobile Device}

Figure 2 depicts the architecture of a smart mobile device for emergency bio-data management based on the personalized emergency policy. The smart mobile device plays a very important role as it receives the bio-data from the body sensors, judges the emergency of bio-data, and forwards the bio-data to the EMS. The smart mobile device consists of a local user information manager, policy manager, and the ISO/IEEE 11073 manager. The local user information manager consists of the user authentication database, the emergency policy database, and the plug-connected ISO/IEEE 11073 agent information database. The local user authentication database stores the authentication information to allow users to access the service. The authentication process is also performed by accessing the emergency policy server. The emergency policy database manages and maintains the personalized emergency policy information of each user or patient. The plug-connected ISO/IEEE 11073 agent database manages the device connection information of the plug-connected or unplug-connected ISO/IEEE 11073 agent. The policy manager consists of patient authentication management, bio device management, emergency bio-data management, and protocol conversion management. The policy manager is addressed in detail in Section 3.4. The ISO/IEEE 11073 manager collects the biodata from ISO/IEEE 11073 agents, which conforms to the ISO/IEEE 11073-20601 standards. The ISO/IEEE 11073 manager consists of a DIM analyzer, service message composer, and the 11073 transport manager [1]. 


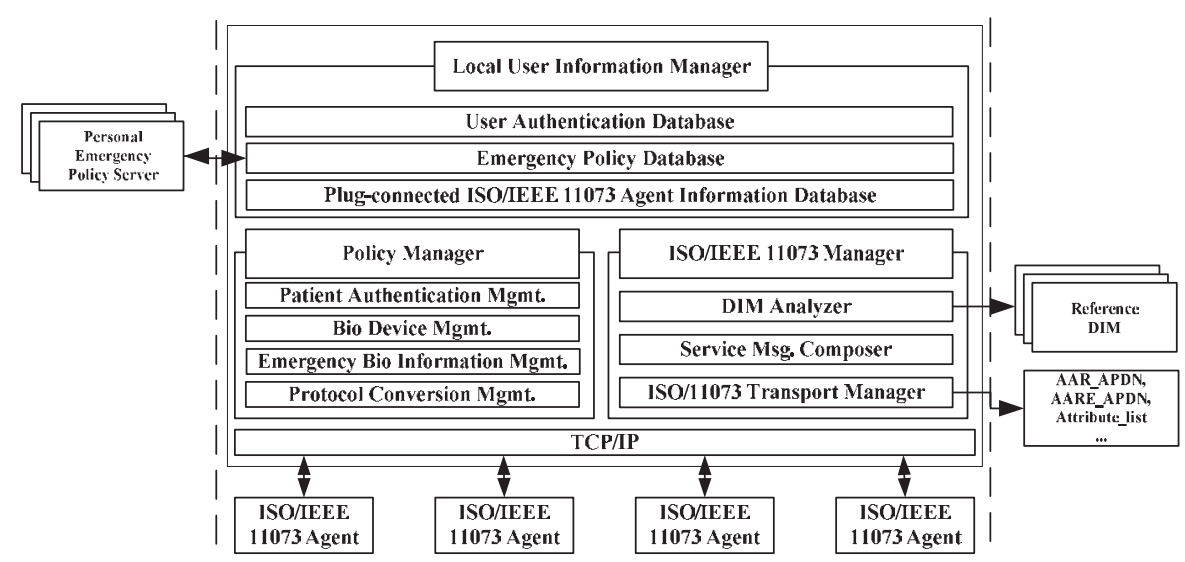

Figure 2. Architecture of the smart mobile device.

\subsection{Architecture of Emergency Management Server}

Figure 3 depicts the architecture of the emergency management server (EMS). The EMS is located between the ISO/IEEE 11073 manager and the healthcare medical server system, and converts messages from ISO/IEEE 11073 PHD data format to HL7 CDA data format, or from HL7 CDA data format to ISO/IEEE 11073 PHD data format in monitoring and delivering the personalized bio-data. As the smartphone usually has constrained capacity in both power and processing capabilities, there is high probability that the emergency notification may not be delivered in time due to the direct conversion operation between ISO/IEEE 11073 standard and HL7 on the smartphone. Furthermore, since the conversion model is evolving, it may be very difficult to upgrade the up-to-date conversion function on smartphone. Moreover, since the HL7 message is heavy and complex, the wireless communication cost from the smart phone to the gateway using HL7 may be high, comparing with that of using ISO/IEEE 11073 message to the gateway.

The EMS consists of CDA Factory, HL7 v2.x Converter, the ISO/IEEE 11073/HL7 CDA interface, the ISO/IEEE 11073 manager, and the bio-data information manager. CDA Factory produces an HL7 CDA document that includes the bio-data collected in the ISO/IEEE 11073 PHD format. The CDA documentation contains all information related to treatment of the bio-data or patient, and is expressed in the XML format of the reference information model (RIM) of HL7 [14]. The object information for comprising CDA includes Act, Participation, Entity, Role, and Act Relationship. CDA Factory is comprised of CDA Profiler, Medical Information Mapper, and CDA Creator. CDA Profiler verifies the inputted CDA document or outputted CDA document. Medical Information Mapper maps the bio-data and meta information received from HL7 v2.x Converter or the ISO/IEEE 11073 manager to the RIM base class of the CDA document architecture.

The CDA document is expressed in the CDA template which is specified in CDA Profiler. Several CDA templates are generated, which collect the bio-data information from the ISO/IEEE 11073 manager. The generated CDA document is verified again in 


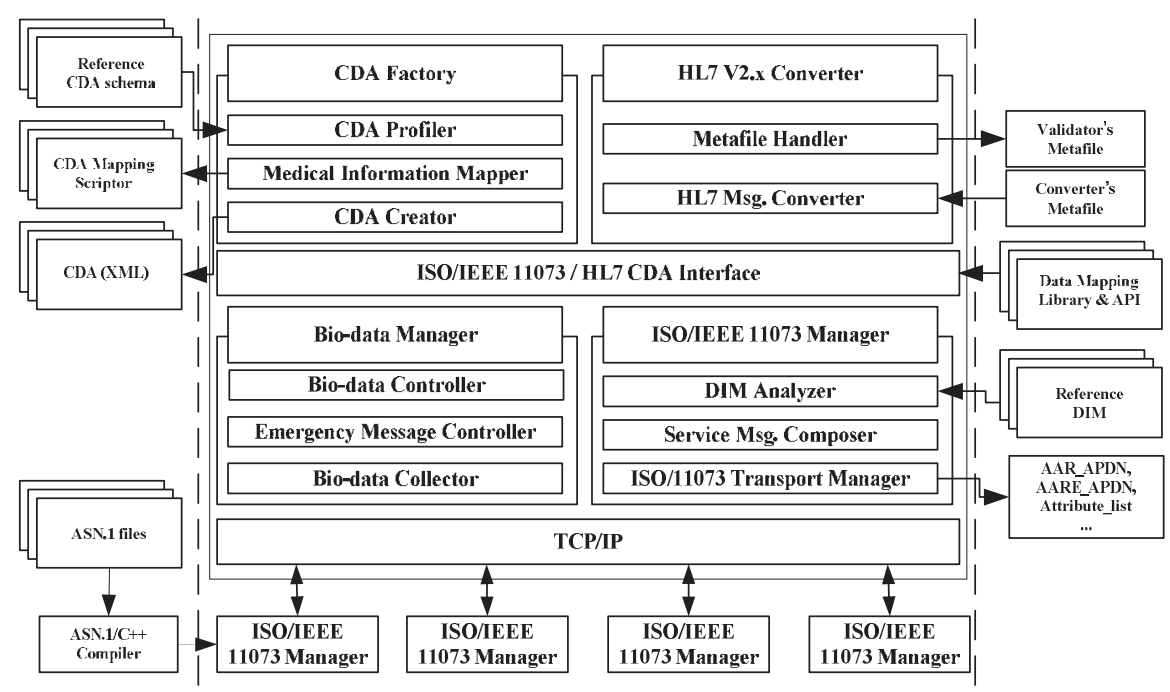

Figure 3. Architecture of emergency management server.

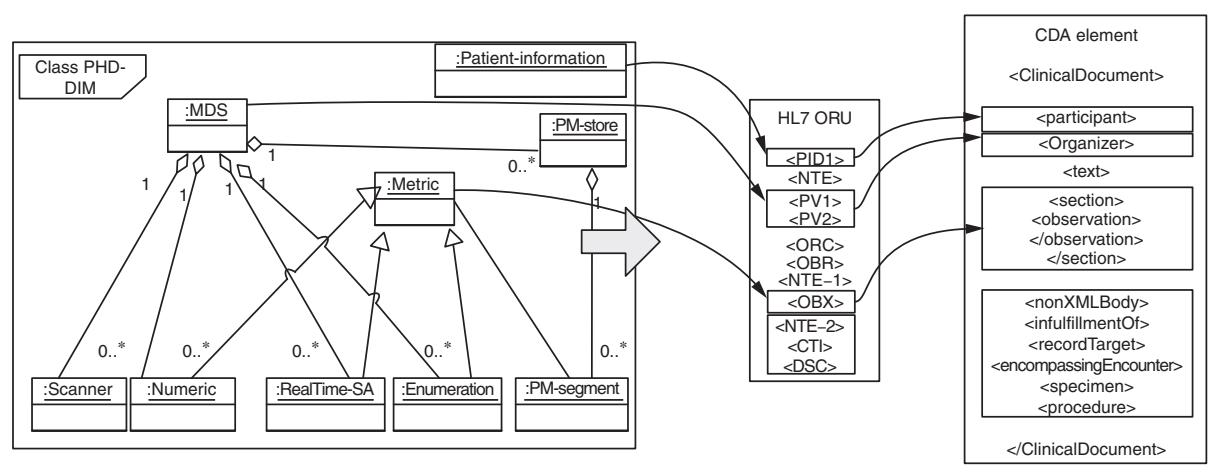

Figure 4. Example of bio-data mapping from ISO/IEEE 11073 PHD to HL7 CDA.

CDA Profiler. HL7 v2.x Converter converts bio-data based on ISO/IEEE 11073 PHD to an HL7 CDA message. HL7 v2.x Converter is comprised of Metafile Handler and HL7 Message Converter. Metafile Handler analyzes the meta data in the HL7 v2.x message, and verifies the meta information. HL7 Message Converter parses the HL7 message after it requests the bio-data from the ISO/IEEE 11073 manager. The bio-data mapping between ISO/IEEE 11073 PHD and HL7 CDA is performed by IEEE11073 PHD/HL7 CDA conversion module.

Figure 4 shows the mapping example for bio-data between ISO/IEEE 11073 DIM and HL7 CDA. The ISO/IEEE 11073 DIM specifies the bio-data from an agent as a set of objects with one or more attributes. It describes both the measurement data to an ISO/IEEE 11073 manager and other control data on the status of the agent. The 
DIM is comprised of zero or more numeric, real-time sample array (RT-SA), enumeration, scanner, or PM-store objects associated with an MDS object as shown in Figure 4. There are zero or more PM-segments that contain persistent metrics associated with a PM-store. Numeric, RT-SA, and enumeration are derived from a common metric base class that contains common and shared attributes. In general, numeric objects represent episodic measurements, RT-SA objects represent continuous samples or wave forms, and enumeration objects represent event annotations. PM-stores along with PM-segments provide a persistent storage mechanism for metrics that are accessed by the manager at a later time. In addition, a scanner object facilitates the reporting of agent-initiated data transfers [1].

The type of HL7 CDA message includes the acknowledgement (ACK), admission, discharge and transfer (ADT), ADT report (ADR), order message (ORM), observational result (ORU), query of results of observation (ORF), and query (QRY). Each message may include several segments which can be included in a message optionally. The type of segment includes message header (MSH), patient identification (PID), observation (OBX), observation request (OBR), patient demographics (PD1), message acknowledgement (MSA), etc. The EMS performs the message conversion from ISO/IEEE 11073 PHD DIM to HL7 CDA. To convert the message format, the ORU message in HL7 CDA and MDS object in ISO/IEEE 11073 PHD DIM are used. The observation result transmitted from ISO/IEEE 11073 PHD manager which included the numeric, RA-SA, and enumeration is mapped to OBX segment in ORU message. HL7 ORU message is converted to HL7 CDA document in order to be transmitted to HL7 system.

The bio-data manager consists of a bio-data controller, an emergency message controller, and a bio-data collector. The bio-data information manager detects an emergency condition based on bio-data received from the body sensors. If an emergency condition in the bio-data is detected, an emergency notification message is created and transmitted to the EMS. The bio-data collector finally transmits the bio-data information from the EMS to the ISO/IEEE 11073 PHD manager. The received bio-data information converts the HL7 CDA message format in accordance with the policy information. The ISO/IEEE 11073 manager performs the collection of the bio-data through communication messages such as GET, SET, ACTION, EVENT REPORT, etc., from the ISO/IEEE 11073 agents. The ISO/IEEE 11073 manager consists of DIM Analyser, Service Msg. Composer, and 11073 Transport Manager. More detailed information about the ISO/IEEE 11073 manager can be found in reference [1], and it is out of the scope of this paper.

\subsection{Message Flow Diagram for Policy-Based Emergency Service Management}

This section describes the message flow diagram for the emergency bio-data management using the personalized emergency policy. Figure 5 shows the message flow diagram for emergency service management using the personalized emergency 


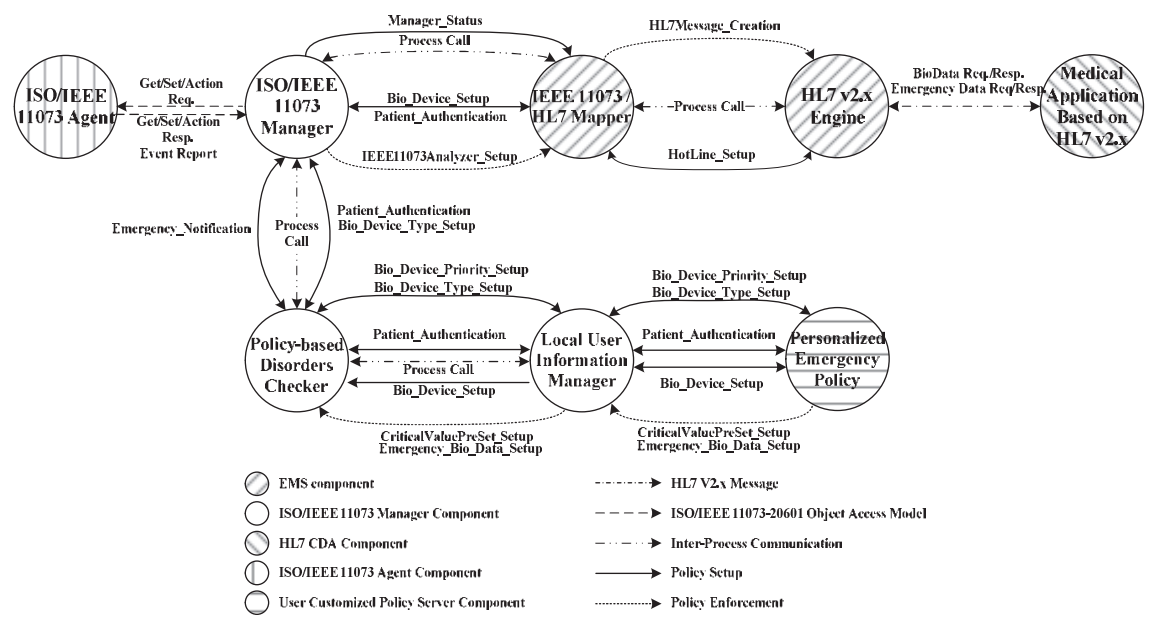

Figure 5. Message flow diagram for policy-based emergency service management.

policy. Table 1 describes the management service for emergency healthcare monitoring. The management service consists of patient authentication management, bio device management, emergency management, and protocol conversion management. As shown in Figure 5, the ISO/IEEE 11073 manager collects the biodata and the connection status of the bio device, from the ISO/IEEE 11073 agent, using GET/SET/ACTION/EVENT REPORT of the ISO/IEEE 11073-20601 standard. As the ISO/IEEE 11073 manager is activated, a Patient_Authentication message is first exchanged between the ISO/IEEE 11073 manager and the personalized emergency policy server in order to authenticate the patient or user. As the ISO/IEEE 11073 agent is newly connected, the ISO/IEEE 11073 manager exchanges messages such as Bio_Device_Type_Setup, Bio_Device_Setup, Bio_Device_Priority_Setup and CriticalValuePreSet_Setup in order to establish emergency judgment process for the bio-data.

The bio device information and bio-data information are stored in an emergency policy database. The ISO/IEEE 11073 manager and ISO/IEEE 11073/HL7 mapper exchange messages such as Bio_Device_setUp, Patient_Authentication, ISO/IEEE 11073 Analyzer_setUp, and Manager_Status in order to transmit the bio-data of the patient to the HL7 CDA application. The critical values are stored in the emergency policy database of the local user information manager in the ISO/IEEE 11073 manager through the CriticalValuePreSet_Setup message. The collected bio-data are judged using the emergency policy database. If a disorder in the bio-data is detected, the policy-based disorder checker generates the process Emergency_Notification. 
Table 1. Management service for emergency healthcare monitoring.

\begin{tabular}{|c|c|c|}
\hline & Policy & Description \\
\hline $\begin{array}{l}\text { Patient } \\
\text { Auth. Mgt. }\end{array}$ & Patient_Authentication & $\begin{array}{l}\text { Delivery of patient authentication information and the } \\
\text { corresponding medical device ID. }\end{array}$ \\
\hline \multirow[t]{4}{*}{$\begin{array}{l}\text { Bio Device } \\
\text { Mgt. }\end{array}$} & Bio_Device_Setup & $\begin{array}{l}\text { Delivery of information of type, model, battery, } \\
\text { voltage, and serial no. of a bio device. }\end{array}$ \\
\hline & Bio_Data_Type_Setup & $\begin{array}{l}\text { Delivery of type information of bio-data, a period of bio-data } \\
\text { measurement of a bio device. }\end{array}$ \\
\hline & CriticalValuePreset_Setup & $\begin{array}{l}\text { Delivery of the preset critical value of each bio-data for } \\
\text { examination at ISO/IEEE } 11073 \text { manager such as critical } \\
\text { range, worming range, normal range, duration of } \\
\text { emergency time of bio-data applied with the personalized } \\
\text { health condition. }\end{array}$ \\
\hline & Emergency_Bio_Data_Setup & $\begin{array}{l}\text { Delivery of the type of bio-data for the emergency } \\
\text { judgment of bio-data such as duration of detection time, } \\
\text { the repetition rate of emergency condition of bio-data. }\end{array}$ \\
\hline \multirow[t]{4}{*}{$\begin{array}{l}\text { Emergency } \\
\text { Mgt. }\end{array}$} & Bio_Device_Priority_Setup & $\begin{array}{l}\text { Delivery of the transmission priority information of each bio } \\
\text { device; i.e., the priority of a bio device is determined by the } \\
\text { health condition of the user. If the patient has cardiovascular } \\
\text { disease, the priority of ECG of various bio-devices is } \\
\text { determined as high. }\end{array}$ \\
\hline & Hotline_Setup & $\begin{array}{l}\text { Delivery of the emergency information and preparation } \\
\text { request for hot line which communicates with physician for } \\
\text { transmission of emergency bio-data. The hot line means that } \\
\text { emergency bio-data should be transmitted to the physician } \\
\text { according to the priority of the bio device. }\end{array}$ \\
\hline & Emergency_Notification & $\begin{array}{l}\text { Event message of the emergency situation of bio-data } \\
\text { through inter-process call. }\end{array}$ \\
\hline & ManagerStatus_Setup & $\begin{array}{l}\text { Delivery of the manager status information such as the list of } \\
\text { the connected ISO/IEEE } 11073 \text { agents. }\end{array}$ \\
\hline \multicolumn{3}{|r|}{ 然 } \\
\hline \multirow[t]{2}{*}{$\begin{array}{l}\text { Conversion } \\
\text { Mgt. }\end{array}$} & IEEE11073Analyzer_Setup & $\begin{array}{l}\text { Delivery of the setup information to be ready } \\
\text { for mapping to HL } 7 \text { message. }\end{array}$ \\
\hline & HL7Message_Creation & $\begin{array}{l}\text { Delivery of bio-data, medical device ID, and patient } \\
\text { information for generation to HL } 7 \text { message. }\end{array}$ \\
\hline
\end{tabular}

After the ISO/IEEE 11073 manager checks the transmission priority of the emergency policy database, the bio-data is transmitted to ISO/IEEE 11073/HL7 Mapper. Finally, the urgent bio-data arrives in the medical application based on HL7.

Figure 6 shows an example of XML schema of Emergency_Bio_Data_Setup part of the emergency management policy [15]. XML element is composed of dataset, section, value, and escape. In Figure 6, Emergency_Bio_Data_Setup message is 


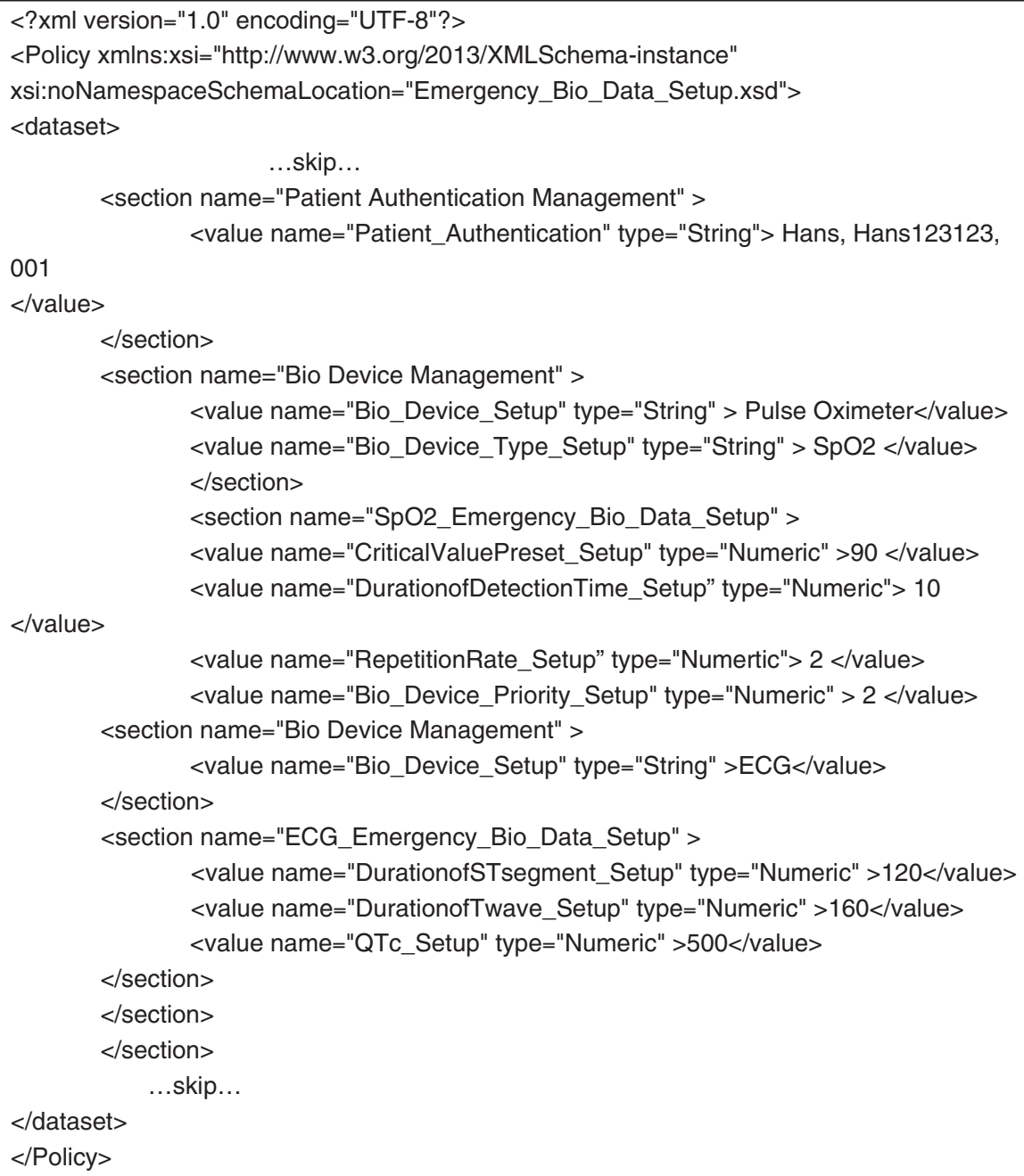

Figure 6. XML schema of Emergency_Bio_Data_Setup part of the emergency management policy.

used to deliver the type of bio-data for the emergency judgment of bio-data such as duration of detection time, the repetition rate of emergency condition of bio-data. To this end, the Emergency_Bio_Data_Setup message includes the patient authentication management policy, bio device management, and emergency management policy. Emergency_Bio_Setup message includes the CriticalValuePreset_Setup, DurationofDetectionTime_Setup, RepetitionRate_Setup, and Bio_Device_Priority_Setup. 
In Figure 6, Emergency_Bio_Data_Setup are shown for SpO2 and ECG data. For determining emergency condition for $\mathrm{SpO} 2$, the guideline descried in the reference [16] is used. Levels of $\mathrm{SpO} 2$ range from 96 to $99 \%$ in healthy individuals. However, when patients have pulmonary or cardiovascular chronic diseases with concomitant common cold or pneumonia, the level of SpO2 may drop rapidly. SpO2 lower than $90 \%$ is defined as acute respiratory failure [16]. When SpO2 drops by 3 to $4 \%$ from its normal level, even if it is not less than $90 \%$, an acute disease may be suspected. For determining emergency condition for ECG data, duration of ST segment, QT dispersion and the T wave morphology can be considered. The ST segment represents the period when the ventricles are depolarized. The T wave represents the recovery of the ventricles. It is known that the normal duration of ST segment is 80 to $120 \mathrm{~ms}$. For QT dispersion, the mean change of QTc interval indicates the degree of risk in heart. QTc is the QT corrected for heart rate. The condition of QTc $>500 \mathrm{~ms}$ indicates a life-threatening signs or symptoms [17]. In Figure 6, we have designed these ECG emergency conditions in ECG_Emergency_Bio_Data_Setup. More detailed analysis on ECG emergency conditions is out of scope of this paper.

\section{IMPLEMENTATION}

\subsection{System Configuration}

Figure 7 shows the system configuration for the implementation of the prototype of the proposed system using the personalized emergency policy. We have used two types of sensors: ECG sensor and $\mathrm{SpO}_{2}$ sensor. We used an HBE-ZigbeX2 device

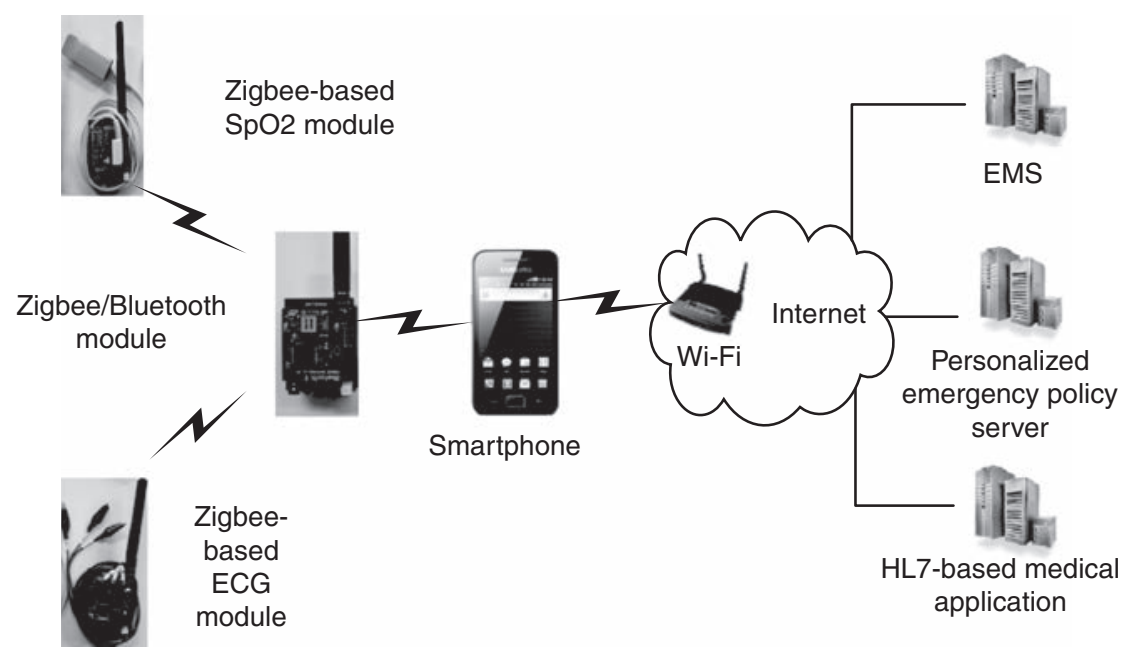

Figure 7. Prototype system configuration of personalized emergency policy-based healthcare monitoring system. 
developed by the HANBACK Company (http://www.Handback.co.kr) in Korea. The data models of ECG and $\mathrm{SpO}_{2}$ complied with ISO/IEEE 11073-10404: 2010 [18] and ISO/IEEE 11073-10406:2012 [19] standards, respectively.

- $\quad$ Server : IBM Server, Apache 2.2, MySQL 5.5

- Smart mobile device : Galaxy S2 (SHWM250K model)-OS version 2.5

- Type of sensor (product standard): Bluetooth module (HBE-ZigbeX2-Bluetooth), $\mathrm{SpO}_{2}$ sensor module (HBE-ZigbeX2- $\mathrm{SpO}_{2}$ ), ECG sensor module

- $\mathrm{SpO}_{2}$ sensor module specification:

- Infrared light sensor: RED: 660nm/Infrared: 920nm

- $\mathrm{SpO}_{2}$ (\%: saturation): $40 \sim 99 \%$

- $\quad$ Pulse rate range: $30 \sim 300 \mathrm{bpm}$

- Pulse accuracy: +2\%@30 300 bpm

- ECG sensor module specification:

- $\quad$ Micro Controller Unit (MCU): ATmega8L

- Type of sensor: Contactless temperature sensor

- $\quad$ Number of leads: 3-lead

- Development language : visual C, MFC, PHP

Usually, bio-data may be collected over a long period of time in order to allow accurate diagnoses and correct treatment by clinicians or remote caregivers. The policy used in the proposed architecture is to provide the efficiency in data transmission, by avoiding the unnecessary traffic on wireless networks. Since the $\mathrm{SpO}_{2}$ data are accurate enough to show the efficacy of the proposed approach, the experiments have been performed by applying the policy in Figure 6, which includes the emergency management policy for $\mathrm{SpO}_{2}$ data. Due to the limited functionality of ECG sensor module used in our experiments, the available data in ECG monitoring may be too simple to be used for clinical application, and will be studied in further work.

\subsection{Healthcare Monitoring System Scenario}

The system operation scenario is as follows: Two types of bio-data, $\mathrm{SpO}_{2}$ and ECG, are measured by the ZigBee-based $\mathrm{SpO}_{2}$ measurement module and ZigBee-based ECG measurement module, respectively. Two authentication procedures, i.e., the patient authentication and the medical device authentication, are implemented. In the patient authentication, patient ID and password are used, and in the medical device authentication, the node ID is used. The authentication procedure is as follows: First, the patient inputs the patient ID and password. The medical device is then connected to the smartphone and the node ID of the medical device is transmitted to the smartphone. Next, the authentication information is requested by the EMS. In response to the request, the smartphone transmits the authentication result, and the authentication information is transferred to the personalized emergency policy server. The smartphone can finally retrieve the personalized emergency policy. Bio-data are transmitted to a smartphone using ZigBee communication module and Bluetooth communication module. 
A smartphone requests the critical policy information with regard to $\mathrm{SpO}_{2}$ and ECG from a policy-based database server. The smartphone monitors the bio-data information. If the bio-data are found to satisfy the critical emergency condition, the smartphone excerpts the specific bio-data information related to the emergency condition based on the personalized emergency policy, and immediately sends an emergency notification message to the EMS. The EMS then converts the ISO/IEEE 11073 message to the corresponding HL7 CDA message, and sends the converted emergency notification message to the HL7-based medical application. Afterwards, thee hot lines may be set up between the EMS and HL7-based medical application, though which the measured emergency bio-data on the Zigbee-based module can immediately arrive at the HL7-based medical application.

\subsection{Implementation Results and Discussion}

Figures 8-10 show the implementation results of the prototype of the proposed system architecture. Real-time bio-data are collected by Zigbee-based ECG and $\mathrm{SpO}_{2}$ bio sensors in accordance with the ISO/IEEE 11073 message format. Figure 8 shows the screen view of ECG/SpO $\mathrm{Sp}_{2}$ bio-data on a smartphone. While the bio-data are being monitored on the smartphone, the emergency condition of the bio-data is detected. Figure 9 shows screen view in HL7-based medical application. The bio-data of ECG and $\mathrm{SpO}_{2}$ in the HL7-based medical application are received from the EMS in HL7 CDA message format.

Figure 10 shows the emergency notification message is sent from EMS to HL7based medical application. This emergency message is generated by the HL7 v2.1 engine. As shown in Figure 9, as the emergency condition of the patient is detected, the emergency notification and bio-data can be transferred to the medical staff.

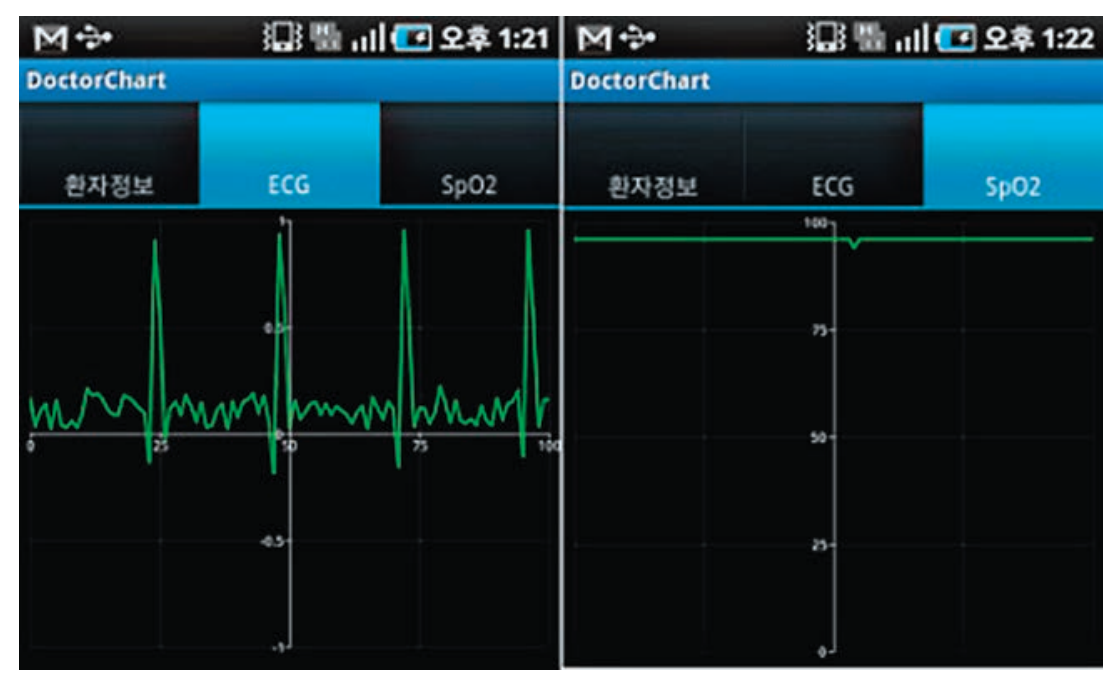

Figure 8. Screen view on smartphone. 


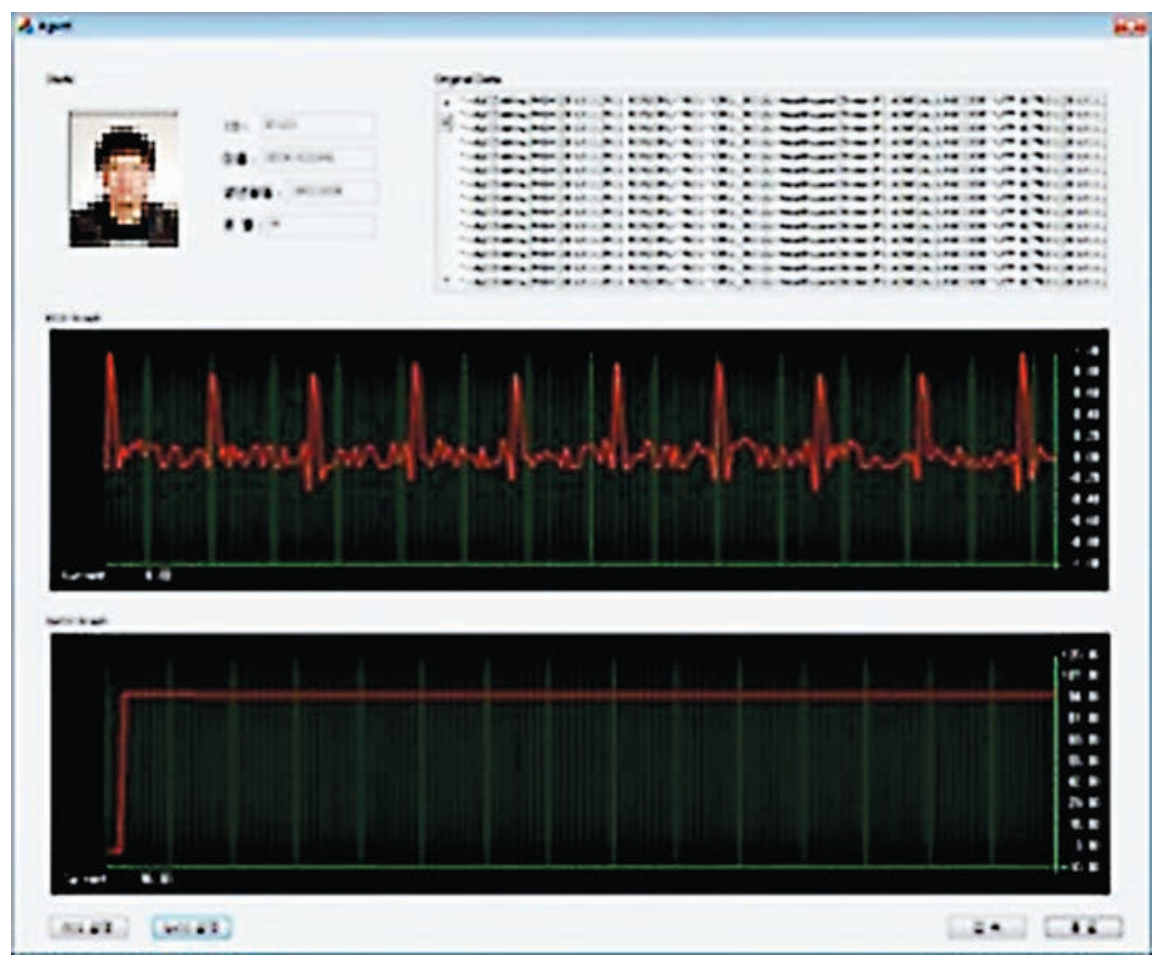

Figure 9. Display view of HL7-based medical application.

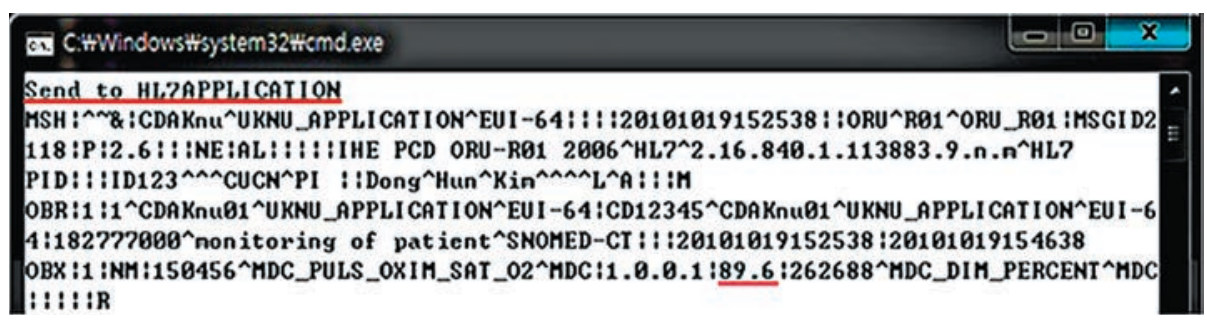

Figure 10. Emergency notification message received from EMS in HL7-based medical application.

\section{CONCLUSION}

In this paper, we have presented an architecture of emergency bio-data management using a personalized emergency policy for mobile healthcare. More specifically, we have designed the management architecture using a personalized emergency policy for mobile healthcare. We have designed an architecture of a smart mobile device for emergency bio-data management based on the personalized emergency policy. The architecture of the emergency management server has also been designed and described 
in detail. One of the key features of the proposed architecture is that the bio-data collected at sensor networks are first represented in ISO/IEEE 11073 standard format, and then converted to HL7 CDA format for reporting to medical staff or physician.

The mapping structure and example for bio-data between ISO/IEEE 11073 PHD DIM and HL7 CDA are described, with message flow diagram for policy-based emergency service management. In addition, the table for specific management service for emergency healthcare monitoring is presented. XML schema of Emergency Bio_Data_Setup part of the emergency management policy is also described in detail. Finally, the prototype of the proposed system has been built to demonstrate the design concept, the service, and functions of the proposed system architecture. Using this system, when an emergency is detected, emergency rescue service can be provided to the patient both accurately and immediately.

\section{ACKNOWLEDGEMENTS}

This research was supported by BK21 Project and Kyungpook National University Research Fund. The authors would like to thank the anonymous reviewers for their valuable comments.

\section{CONFLICT OF INTEREST}

The authors indicated no potential conflicts of interest.

\section{REFERENCES}

[1] ISO/IEEE 11073-20601 Standard for Health Informatics - Personalized health device communication Application profile - Optimized exchange protocol-ISO/IEEE 11073-20601. The Institute of Electrical and Electronics Engineers. 2008.

[2] HL7 Clinical Document Architecture (CDA), Release 2.0, Health Level 7, Apr. 2005.

[3] Shen X, Emerging Technologies for e-healthcare. IEEE Network, 2012, 26(5):2-3.

[4] Franklin S, Larson M, Khan S, Wong N, Leip E, Kannel W, Levy D. Does the Relation of Blood Pressure to Coronary Heart Disease Risk Change With Aging?: The Framingham Heart Study. American Heart Association, 2001, 103: 1245-1249.

[5] Skurnick J, Aladjem M, Aviv A. Sex Differences in Pulse Pressure Trends With Age Are CrossCultural. American Heart Association, Jan 2010, 55(1): 40-47.

[6] Hoekema R, Uijen G, Oosterom A. Geometrical aspects of the interindividual variability of multilead ECG recordings. IEEE Trans. Biomed. Eng., May 2001, 48(5): 551-559.

[7] Wei J, Kong G, Block-Based Neural Networks for Personalized ECG Signal Classification. IEEE Transactions on Neural Networks, Nov. 2007, 18(6):1750-1761.

[8] Trigo JD, Chiarugi D, Alesanco F, et al. Interoperability in digital electrocardiography: harmonization of ISO/IEEE x73-PHD and SCP-ECG. IEEE Trans. on Info. Tech. Biomed, 2010, 14(6): 1303-17.

[9] Yao J, Warren S. Applying the ISO/IEEE 11073 Standards to Wearable home Health Monitoring Systems. Journal of Clinical Monitoring and Computing, Jan. 2006, 19(6): 427-436.

[10] Mense A, Sauermann S, Gerbovics F, Frohner M, Wahl H, Pucher R. "Healthy interoperability": A standard based framework for integrating personal monitoring and personal health device data into medical information systems. Journal on Information Technology in Healthcare, 2009, 7:214-221.

[11] Yuksel M, Dogac A. Interoperability of Medical Device Information and the Clinical Applications: An HL7 RMIM based on the ISO/IEEE 11073 DIM. IEEE Transactions on Information Technology in Biomedicine, 2011, 1(15): 557-566. 
[12] Yang M, Chronaki C, et al., Guideline-driven telemonitoring and follow-up of cardiovascular implantable electronic devices using IEEE 11073, HL7 \& IHE profiles. Proc. of IEEE Engineering Medical Biology Society, 2011, 3192-6.

[13] Lim J, Park C, Pak S. Home Healthcare Settop-box for Senior Chronic Care using ISO/IEEE 11073 PHD standard. IEEE Engineering in Medicine and Biology Society (EMBC), 2010, 216-219.

[14] Robert H, Liora A, Sandy B, Calvin B, Fred M, Paul V, Amnon S. HL7 Clinical Document Architecture, Release 2. J. Am. Med. Inform. Assoc., 2005, 13(1): 30-39.

[15] BIOTRONIK, BIOTRONIK IEEE 11073-10103 XML Structure, Nov. 2010.

[16] How to Read SpO2: Basic understanding of the pulse oximeter. Konica Minolta Sensing, Inc. 2006

[17] Morganroth J, Shah RR, Scott JW. Evaluation and Management of Cardiac safety using the Electrocardiogram in Oncology Clinical Trials: Focus on Cardiac Repolarization (QTc Interval). Clinical Pharmacology \& Therapeutics, Dec. 2009, 87(2):166-74 9.

[18] ISO/IEEE 11073-10404:2010 Health Informatics Đ Personal Health Device Communication Đ Device Specialization $Đ$ Pulse Oximeter, The Institute of Electrical and Electronics Engineers, New York, USA, April 2008, 1-61.

[19] ISO/IEEE 11073-10406:2012 Health Informatics - Personal Health Device Communication - Part 10406: Device Specialization - Basic electrocardiograph (ECG), The Institute of Electrical and Electronics Engineers, New York, USA, 2012.

[20] Trigo JD, Martinez I, Alesanco A, Kollmann A, Escayola J, Hayn D. et al. An integrated healthcare information system for end-to-end standardized exchange and homogeneous management of digital ECG formats. IEEE Transaction Information Technology Biomed, Jul 2012, 16: 518-29. 



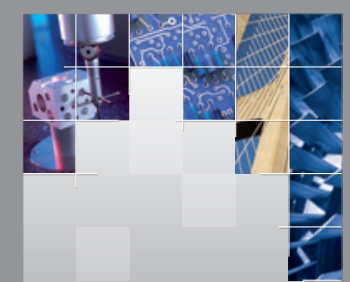

\section{Enfincering}
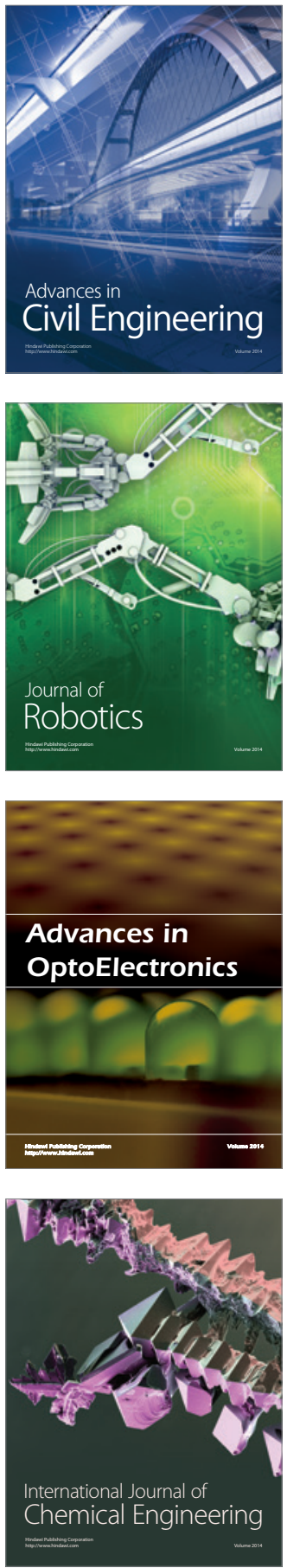

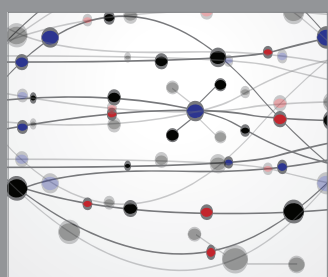

The Scientific World Journal

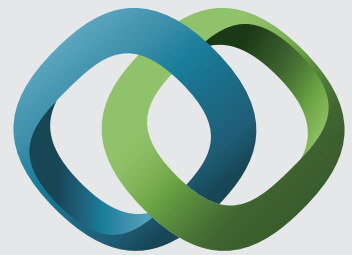

\section{Hindawi}

Submit your manuscripts at

http://www.hindawi.com
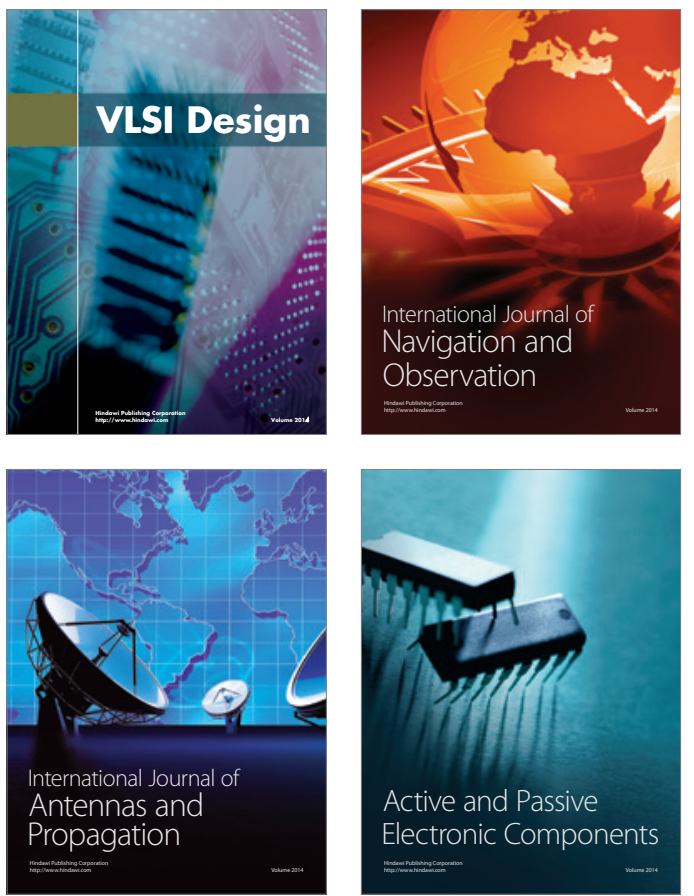
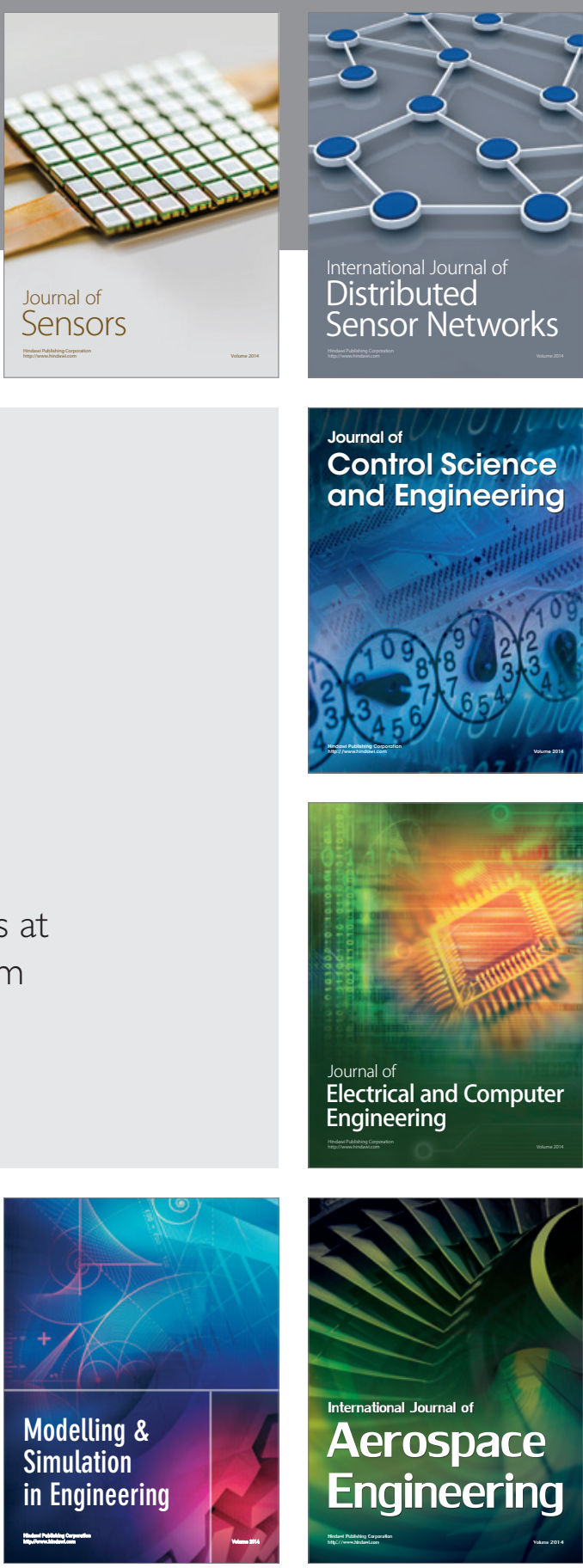

International Journal of

Distributed

Sensor Networks

Journal of

Control Science

and Engineering
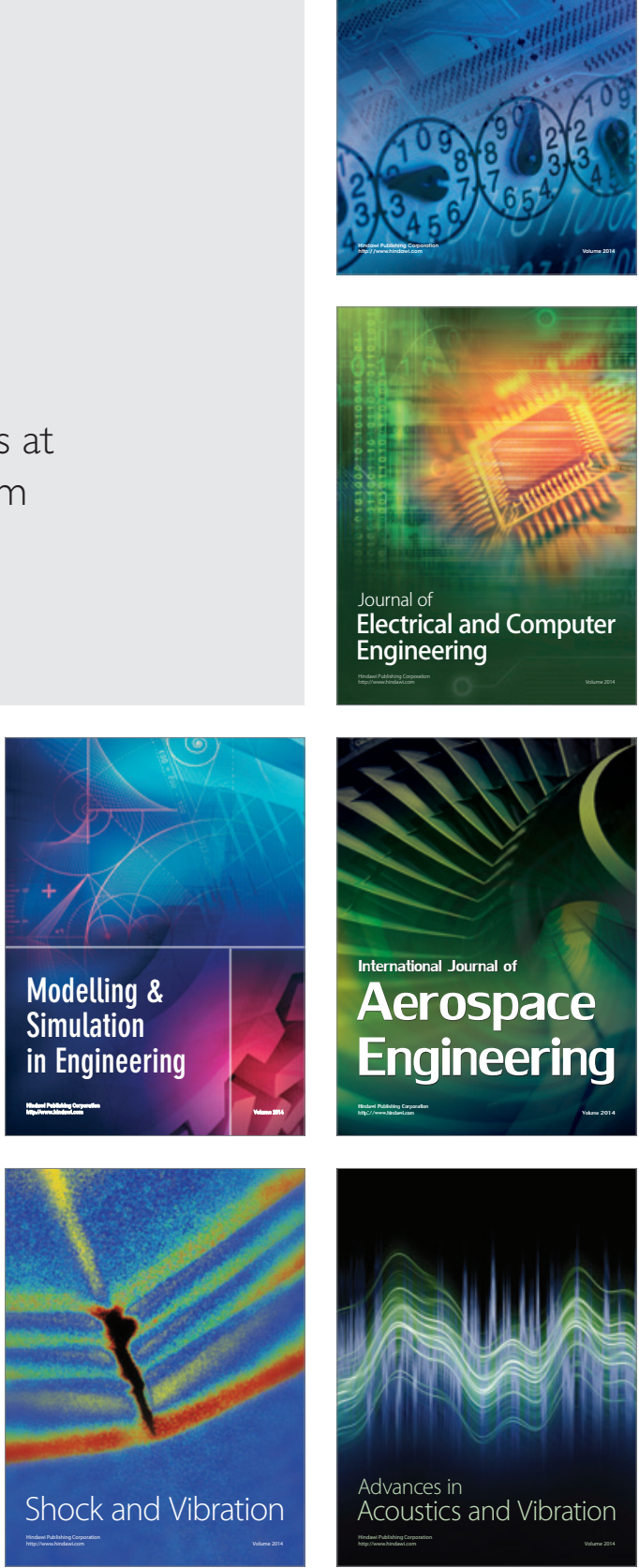